\title{
Blastic Plasmacytoid Dendritic Cell Neoplasm on the Scalp
}

\author{
Sawako Fukushi Taku Fujimura Setsuya Aiba \\ Department of Dermatology, Tohoku University Graduate School of Medicine, \\ Sendai, Japan
}

\section{Key Words}

Blastic plasmacytoid dendritic cell neoplasm $\cdot$ CD56 $\cdot$ Hematopoietic neoplasm

\begin{abstract}
We describe a 75-year-old Japanese woman with blastic plasmacytoid dendritic cell neoplasm. On her first visit, there were multiple areas of disseminated, elastic hard nodules with multicentric purpura on her scalp. Histopathologically, there were atypical large lymphocytes densely infiltrated through the upper dermis to subcutaneous tissue. Immunohistochemical staining revealed that these infiltrated lymphocytes were CD4, CD56 and vimentin positive. Although we administered several courses of intensive chemotherapy soon after the diagnosis, the patient died 5 months after the first visit.
\end{abstract}

\section{Introduction}

Blastic plasmacytoid dendritic cell neoplasm (BPDCN) is recognized as an independent entity in the World Health Organization (WHO) 2008 classification for tumors of hematopoietic and lymphoid tissues [1] and is thought to be derived from the precursors of plasmacytoid dendritic cells [2]. Recently, several reports suggested that, in patients with BPDCN, the skin is the first organ to become clinically involved. Indeed, various clinical features of the skin area have been reported [2-4]. Typically, the involved skin areas of BPDCN are described as showing solitary or multifocal bruiselike papules, nodules or plaques [2]. Moreover, BPDCN clinically mimicking cutaneous lupus erythematosus has also been reported [5]. The disease consistently runs an aggressive course and most patients die within the first year after the diagnosis is established [3]. Because previous reports indicate that early chemotherapy might show a temporary effect in some rare patients and even prolong the survival rate, the accurate and rapid diagnosis for this disease is indispensable $[3,4]$. 


\section{Case Report}

A 75-year-old Japanese woman visited us with a one-month history of nodules developing on her scalp. On her first visit to our hospital, physical examination disclosed multiple areas of disseminated, elastic hard nodules with multicentric purpura on her scalp (fig. 1). Histopathologically, there were atypical large lymphocytes densely infiltrated through the upper dermis to subcutaneous tissue with minimal involvement of the overlying epidermis (fig. 2a). Immunohistochemical staining revealed that these infiltrated lymphocytes were CD4, CD56 (fig. 2c, d) and vimentin positive, CD3, CD5, CD7, CD8 and CD20 negative. Flow cytometry of the bone marrow lymphocytes revealed cellular characteristics as follows: CD4+, CD56+, CD1-, CD2-, CD3-, CD5-, CD7-, CD8-, CD10-, CD14-, CD19-, CD20-, CD33-, CD34-. From the above data, we diagnosed this patient as having blastic plasmacytoid dendritic cell neoplasm. We first administered THP-COP therapy (pirarubicin, cyclophosphamide, oncovin and prednisone). After this intensive chemotherapy, the nodules on the scalp regressed temporarily; however, two weeks later, the tumors were rapidly increasing. Thus, we selected ATriplev therapy (cytarabine, etoposide, vincristine and vinblastine) and DeVIC therapy (carboplatin, ifosfamide, etoposide, dexamethasone) but there was no effect on the tumor growth. Four months after the administration of intensive chemotherapy, the patient died before restarting chemotherapy.

\section{Discussion}

We describe a case of BPDCN on the scalp. Cutaneous involvement is often the first manifestation of this disorder, and the lesions have specific features, presenting as solitary or multifocal bruise-like nodules and plaques [6]. Thus, dermatologists need to be familiar with its clinical features and prognosis.

BPDCN is a rare and highly aggressive lymphoma. It was formally known as blastic natural killer (NK) lymphoma, or agranular CD4+CD56+ hematodermic neoplasm, and accounts for about $0.7 \%$ of all skin and primary malignant lymphoma [7]. The etiology of BPDCN is still unclear. The main clinical features of BPDCN are infiltration of the skin and subsequent involvement of bone marrow and lymph nodes. Its typical pathologic characteristics are uniformly distributed, dense, medium-sized lymphocytes in the dermis layer. Tumor cells are similar in appearance, with a medium-sized, round, or irregular nucleus, and the nucleolus is not obvious [6,7]. Typical immunohistochemistry results include positive for CD4, CD56, negative for CD3, CD20, myeloperoxidase, and CD33. This type of lymphoma is highly aggressive and most patients with BPDCN die within a short period of time after diagnosis $[4,8]$. Conventional chemotherapy is ineffective. Although early chemotherapy may show a temporary effect in some rare patients, the disease often recurs soon afterward [4]. Early intensive chemotherapy may yield a better outcome [3, 4]. However, the chance of post-treatment recurrence is much higher than with other malignant lymphomas.

In our present case, systemic spread of the tumor was detected within the first month. Thus, even though we administered intensive chemotherapy soon after the diagnosis, the patient died 5 months after her first visit to our clinic. Therefore, dermatologists need to be familiar with the clinical features of BPDCN to diagnose this disease immediately. 


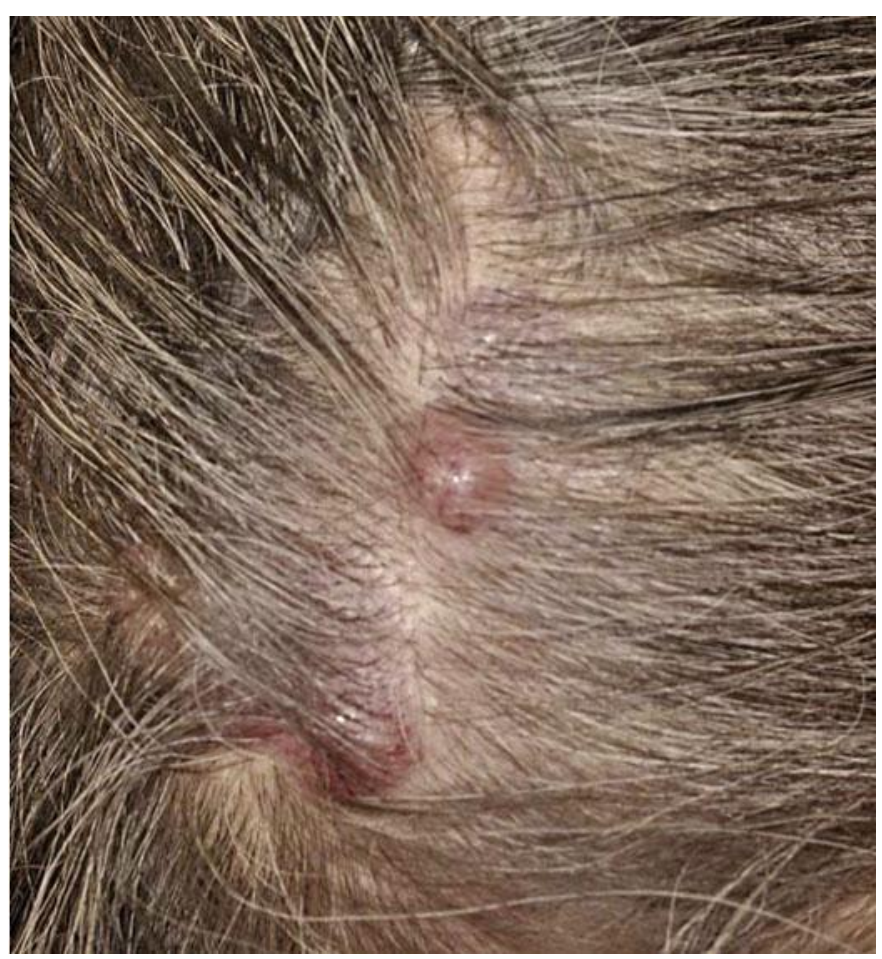

Fig. 1. Multiple areas of disseminated, elastic hard nodules with multicentric purpura on the patient's scalp. 

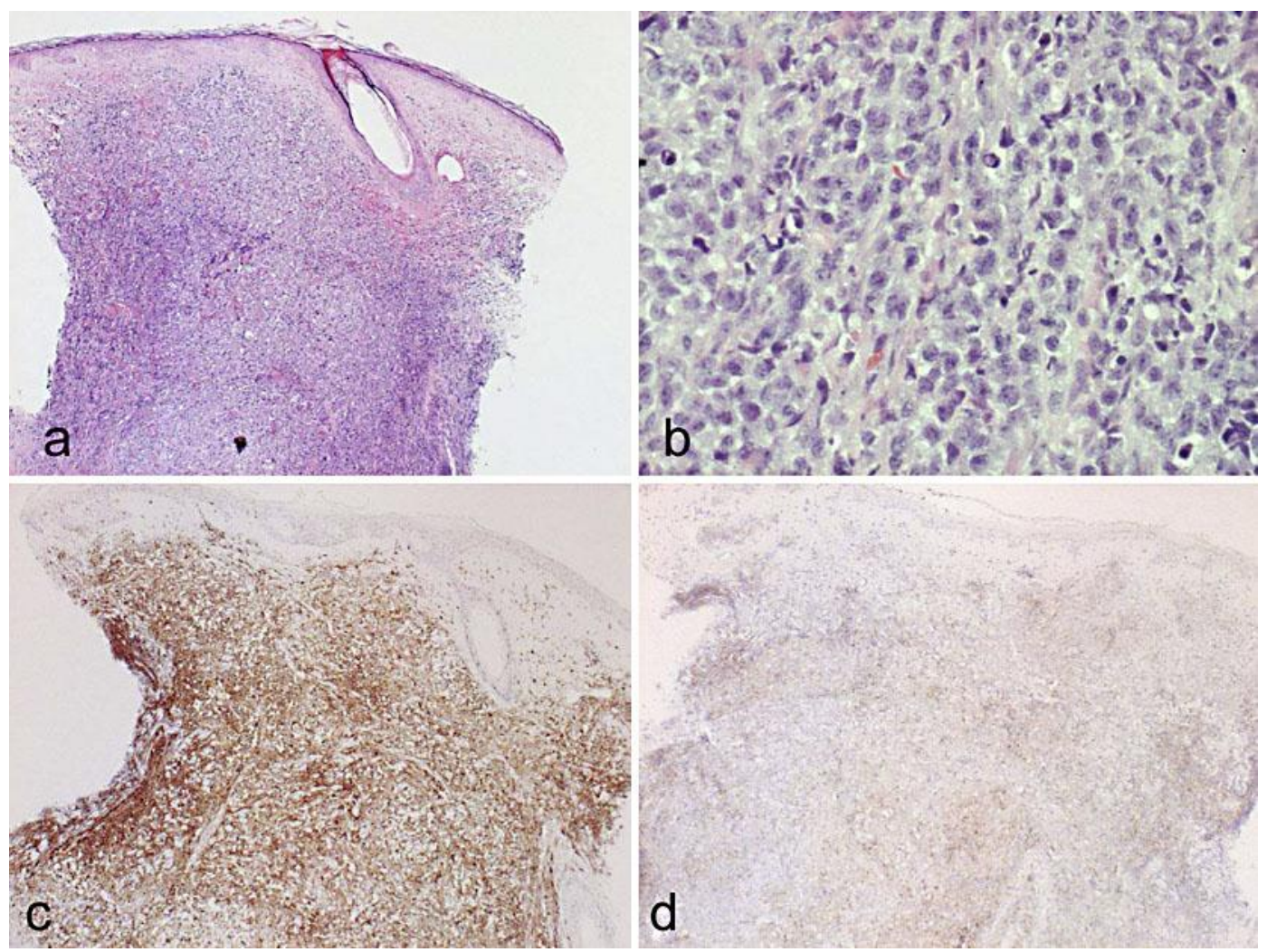

Fig. 2. Atypical large lymphocytes densely infiltrated through the upper dermis to subcutaneous tissue with minimal involvement of the overlying epidermis (a; H\&E staining, original magnification $\times 50$ ). Median-sized monomorphous cells with irregular nuclei were infiltrating (b; H\&E staining, original magnification $\times 400$ ). Immunohistochemical staining for CD4 (c) and CD56 (d; original magnification $\times 50)$.

\section{References}

1 Swerdlow SH, Campo E, Harris NL, Jaffe ES, Pileri SA, Stein H, Thiele J, Vardiman JW: WHO Classification of Tumours of Haematopoietic and Lymphoid Tissues. 4th ed. Lyon, France: IARC Press; 2008. WHO Classification of Tumours; vol 2, pp 145-147.

-2 Petrella T, Meijer CJ, Dalac S, Willemze R, Maynadie M, Machet L, Casasnovas O, Vergier B, Teitell MA: TCL1 and CLA expression in agranular CD4/CD56 hematodermic neoplasms (blastic NK-cell lymphomas) and leukemia cutis. Am J Clin Pathol 2004;122:307-313.

-3 Hallermann C, Middel P, Griesinger F, Gunawan B, Neumann C: CD4+ CD56+ blastic tumor of the skin: cytogenetic observations and further evidence of an origin from plasmocytoid dendritic cells. Eur J Dermatol 2004;14:317-322.

-4 Petrella T, Bagot M, Willemze R, Beylot-Barry M, Vergier B, Delaunay M, Meijer CJ, Courville P, Joly P, Grange F, De Muret A, Machet L, Dompmartin A, Bosq J, Durlach A, Bernard P, Dalac S, Dechelotte P, D'Incan M, Wechsler J, Teitell MA: Blastic NK-cell lymphomas (agranular CD4+CD56+ hematodermic neoplasms): a review. Am J Clin Pathol 2005;123:662-675.

5 Chang HJ, Lee MD, Yi HG, Lim JH, Shin JH, Choi SJ, Moon Y, Nahm CH, Kim CS: A case of blastic plasmacytoid dendritic cell neoplasm initially mimicking cutaneous lupus erythematosus. Cancer Res Treat 2010;42:239-243.

-6 Cheng J, Zhou J, Qin D, Xu S, Yan X: Blastic plasmacytoid dendritic cell neoplasm. J Clin Oncol 2011;29:e27-e29.

7 Xue R, Wu T, Pan H, Gu, Y, Yang B, Chen Y, Qiu J: A case of cutaneous blastic plasmacytoid dendritic cell neoplasm. Acta Derm Venereol 2010;90:645-646.

8 Margo CM, Porcu P, Schaefer J, Erter JW, Furman RR, Shitabata PK, Crowson AN: Cutaneous CD4+CD56+ hematologic malignancies. J Am Acad Dermatol 2010;63:292-308. 